\title{
A Rare Presentation of Euthyroid Orbitopathy in Papillary Thyroid Carcinoma
}

Pankaj Chaturvedi, Abhishek Vaidya, Anup Sunil Tamhankar

\begin{abstract}
Ophthalmopathy is one of the known presenting features of Grave's disease. Its due to development of antithyroid receptor antibodies. Rarely it may be associated with thyroid malignancies developing in the Grave's nodule. There are few case reports of patients with coexisting thyroid associated orbitopathy (TAO) and thyroid carcinoma. Sometimes it may be the presenting symptom of an occult thyroid malignancy. Its still rarer if the patient is euthyroid on presentation. It is associated with low titers of antithyroid receptor antibodies. Here we present a very rare case of symmetrical nonmetastatic orbitopathy in a euthyroid papillary carcinoma of thyroid.
\end{abstract}

Key message: Its important to rule out coexisting thyroid cancer in patient presenting with orbitopathy even if euthyroid. Orbitopathy may regress after thyroidectomy. Association of thyroid cancer, autoimmunity and orbitopathy is still to be proven due to lack of large case series.

Keywords: Thyroid neoplasms, Euthyroid goiter, Exophthalmos.

How to cite this article: Chaturvedi $\mathrm{P}$, Vaidya $\mathrm{A}$, Tamhankar AS. A Rare Presentation of Euthyroid Orbitopathy in Papillary Thyroid Carcinoma. Int J Head Neck Surg 2013;4(3):145-147.

Source of support: Nil

Conflict of interest: None declared

\section{INTRODUCTION}

Thyroid orbitopathy is one of the known presenting features of Grave's disease. Its due to deposition of excessive soft tissue in periorbital region and eyelids as well as overactivity of levator palpebrae superioris due to sympathetic overdrive. Its due to development of antithyroid receptor antibodies. Rarely it may be associated with thyroid malignancies developing in the Grave's nodule. There are only scattered case reports of patients with coexisting thyroid associated orbitopathy (TAO) and thyroid carcinoma. ${ }^{1,2}$ Sometimes it may be the presenting symptom of an occult thyroid malignancy. Its still rarer if the patient is euthyroid on presentation.

Here we present a very rare case of symmetrical nonmetastatic orbitopathy in a euthyroid papillary carcinoma of thyroid.

\section{CASE REPORT}

A 35-year-old lady complained of neck swelling since 2 years slowly increasing in size and protrusion of botheyeballs, since 8 to 10 months. There were no symptoms suggestive of hyperthyroidism. On examination, patient had bilaterally enlarged firm thyroid gland nodules with bilateral level II, III and IV lymph nodes. Bilateral symmetrical proptosis was present. Lid lag and lid retraction were present. Stellwag's sign (staring looks and infrequent blinking) was apparent (Fig. 1). Sonography showed hypoechoic, heterogenous nodule in left thyroid lobe $4 \times 3.5 \mathrm{~cm}$ with increased vascularity. It had irregular borders with loss of 'halo sign' from surrounding parenchyma. Left level III hypoechoic lymph nodes were noted with loss of fatty hilum. FNAC of the thyroid nodule and lymph nodes revealed characteristic Orphan Annie nuclei of papillary thyroid carcinoma. Patient was euthyroid on blood investigations. She underwent total thyroidectomy and bilateral level II, III, IV and V neck dissection. Final histopathological report showed welldifferentiated papillary thyroid carcinoma without extrathyroidal extension (Fig. 2). Nodal metastases were present (cT2cN1b). Patient was investigated for ophthalmopathy. CT scan showed bilaterally symmetrical, diffuse involvement of extraocular muscles without any evidence of metastases. She was found to have antithyroid receptor antibodies positive. Patient was given suppressor dose of thyroxin. Under steroid cover she was given radioiodine. There was no worsening of orbitopathy. It improved over a period of 2 months. Patient is disease free at present without any evidence of ophthalmopathy at the end of 2 years of follow-up.

Methods: Search was made in World English literature with help of PubMed, Google-search. Search terminologies used were euthyroid, orbitopathy, thyroid carcinoma.

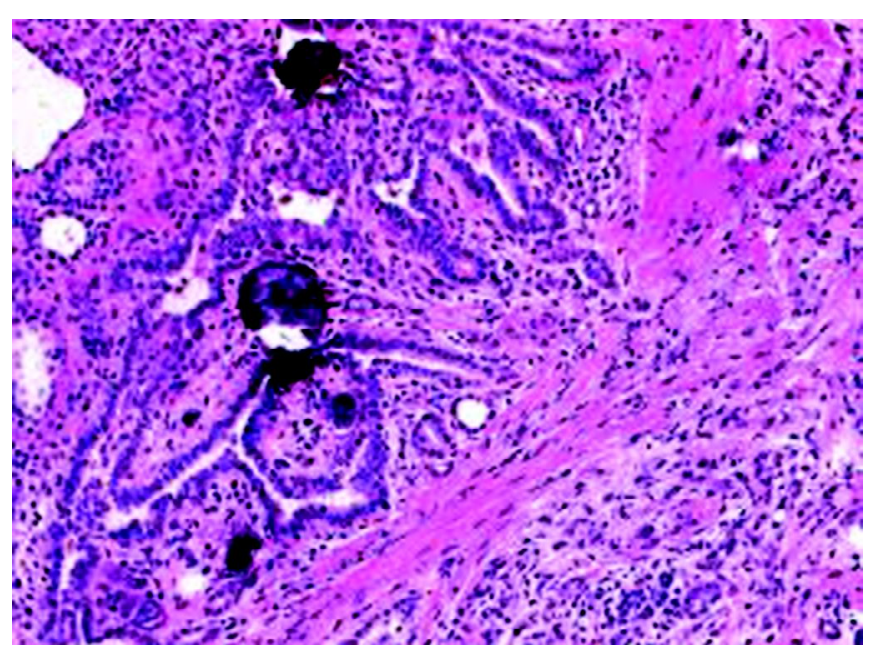

Fig. 1: Bilateral exophthalmos 


\section{DISCUSSION}

Grave's disease is an autoimmune hyperthyroidisminvolving various antibodies directed against thyroid tissue. TAO is clinically evident in 25 to $50 \%$ them. Few studies have shown evidence of common autoantigens between the orbital tissue and thyroid gland. ${ }^{3}$ Recent studies suggest that there is autoantibody involvement in TAO. ${ }^{4}$ Antithyroglobulin antibody and TSI levels and were reported to be higher in patients with a severe course of TAO compared with those with a mild course. This has given rise to thought process of role of TSH receptor antibody as initiating and propagating factor in orbitopathy. ${ }^{4}$ Graves' ophthalmopathy is very rare in euthyroid patients. ${ }^{5}$ Few reports state that the prevalence of TSH receptor antibodies in euthyroid ophthalmopathy exceeds $90 \% .^{5,6}$ One of such studies revealed that TSH receptor antibody titers are usually low, and not associated with the severity of ophthalmopathy in euthyroid Graves' disease. ${ }^{6}$ However, autoantibodies to TSH receptor might have a role in the pathogenesis of ophthalmopathy, even if patients are in a euthyroid state.

Prevalence of incidental microcarcinoma in thyroidectomy specimens of Graves disease ranges from 0.3 to $16.6 \%$ in various studies. ${ }^{7}$ Some studies have shown that patients of Grave's disease with thyroid cancer do better. ${ }^{8}$ On the contrary few others have reported contradictory findings. ${ }^{2}$ However, there is no established relationship between ophthalmopathy, thyroid function, and the biologic behavior of the cancer, when TAO is accompanied by thyroid cancer. It is unclear whether the thyroid cancer cells would elicit autoimmunity in orbital tissues. Also there is no evidence that thyroid autoimmunity might have stimulated thyroid cancer growth. The causal association between thyroid cancer and TAO is difficult to demonstrate in small case series.

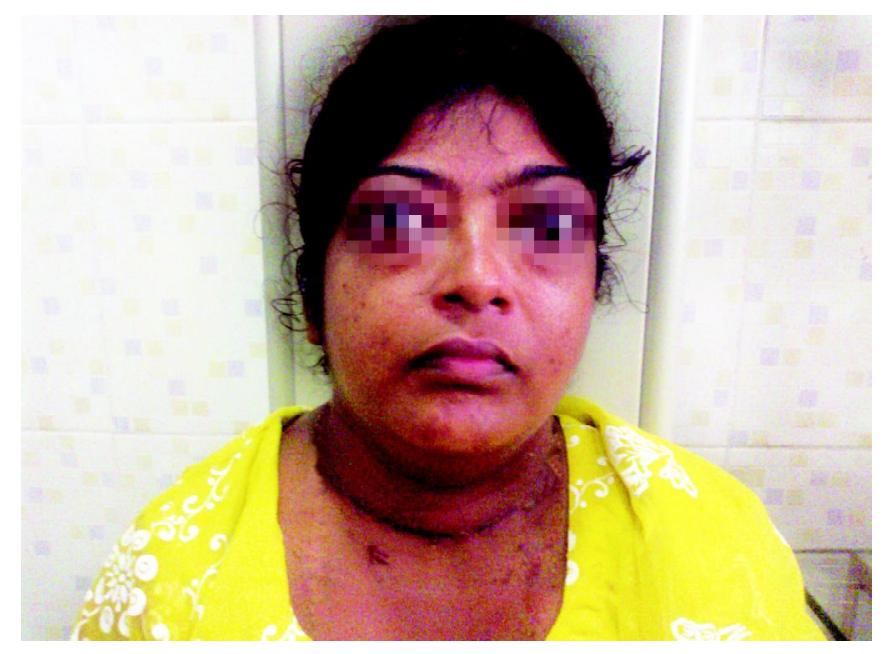

Fig. 2: Psammoma bodies in PTC
Baldys-Waligórska et al reported a case of papillary thyroid cancer in Graves' nodule. Patient developed orbitopathy post-thyroidectomy. Radioiodine was given postoperatively. Though radioiodine is supposed to worsen orbitopathy, in this case it was not found. This may be due to prior removal of thyroid (antigenic source). ${ }^{9}$

Yoon et al presented a review of five cases with euthyroid orbitopathy who were found to have papillary microcarcinoma on investigations. The cases in this review had no clinically apparent goiter. These patients had low titers of thyroid binding immunoglobulin positive. This was a retrospective review and nocausal associations could be established between orbitopathy, autoimmunity and thyroid binding immunoglobulins. Authors emphasized the need of more vigilant work up of patients presenting with orbitopathy alone and asked to do a routine sonongraphy of the neck to pick up any coexisting thyroid malignancies at an early stage. ${ }^{10}$

To our knowledge, present case is the first in the English literature to have euthyroid ophthalmopathy in a case of manifest thyroid malignancy. An interesting observation regarding the present case was that the only abnormality in serum testing was low-titer positive TSH receptor antibody levels at the time of the presentation. This was a unique presentation of orbitopathy in carcinoma thyroid in euthyroid state, which improved after removal of the gland. Our case differs from that of the one reported in the literature in having clinically apparent thyroid malignancy as the presenting complaint rather than orbitopathy itself. So this opens up a clue to possible association of autoimmunity of Grave's ophthalmopathy and thyroid cancer. Obviously this being a single case report further studies and evaluation of similar cases will be required to establish this association. Physician should also take into consideration the possibility of coexisting orbitopathy in a case of thyroid malignancy even though patient is euthyroid. This will prevent drastic consequences of radioiodine on orbitopathy if steroids are started before it. Also as a corollary, a clinician is least likely to expect a malignancy in patient presenting with euthyroid Grave's ophthalmopathy. This warrants more liberal use of imaging and guided biopsies in such cases so as to pick up these cancers at an earlier stage.

\section{REFERENCES}

1. Berg G, Andersson T, Sjodell L, et al. Development of severe thyroid-associated ophthalmopathy in a patient with disseminated thyroid cancer treated with recombinant human thyrotropin/ radioiodine and retinoic acid. Thyroid 2005;15:1389-94.

2. Cobin RH. Thyroid carcinoma and Graves' disease. Endocr Pract 2000;6:264-67.

3. Jyonouchi SC, Valyasevi RW, Harteneck DA, et al. Interleukin6 stimulates thyrotropin receptor expression in human preadipocyte fibroblasts from patients with Graves ophthalmopathy. Thyroid 2001;11:929-34. 
4. Eckstein AK, Plicht M, Lax H, et al. Thyrotropin receptor autoantibodies are independent risk factors for Graves ophthalmopathy and help to predict severity and outcome of the disease. J Clin Endocrinol Metab 2006;91:3464-70.

5. Khoo DH, Eng PH, Ho SC, et al. Graves ophthalmopathy in the absence of elevated free thyroxine and triiodothyronine levels: Prevalence, natural history, and thyrotropin receptor antibody levels. Thyroid 2000;10:1093-100.

6. Kashiwai T, Tada H, Asahi K, et al. Significance of thyroid stimulating antibody and long-term follow-up in patients with euthyroid Graves disease. Endocr J 1995;42:405-12.

7. Farbota LM, Calandra DB, Lawrence AM, Paloyan E. Thyroid carcinoma in Graves disease. Surgery 1985;98:1148-53.

8. Kikuchi S, Noguchi S, Yamashita H, et al. Prognosis of small thyroid cancer in patients with Graves disease. Br J Surg 2006; 93:434-39.

9. Baldys-Waligórska A, Golkowski F, Sokolowski G, BuziakBereza M, Skalski M, Hubalewska-Dydejczyk A. A case of Graves-Basedow disease with orbitopathy and papillary thyroid cancer. Endokrynol Pol 2008 Nov-Dec;59(6):516-20.
10. Yoon JS, Lew H, Park JS, Nam KH, Lee SY. Papillary thyroid carcinoma with thyroid-associated orbitopathy in a euthyroid state. Ophthalmic Plastic Reconstruct Surg 2007;23(3):187-91.

\section{ABOUT THE AUTHORS}

\section{Pankaj Chaturvedi}

Professor, Department of Head and Neck Surgical Oncology Tata Memorial Centre, Mumbai, Maharashtra, India

\section{Abhishek Vaidya}

Senior Resident, Department of Head and Neck Surgical Oncology Tata Memorial Centre, Mumbai, Maharashtra, India

\section{Anup Sunil Tamhankar (Corresponding Author)}

Senior Resident, Department of Surgical Oncology, Tata Memorial Centre, Mumbai, Maharashtra, India, Phone: 7620407925, e-mail: tamhamkaranup@gmail.com 